\title{
Paperless medical clerking: are we still documenting what matters?
}

\author{
Authors: Ahmed El-Masry, Pete Kelly, Shaznin Visanji," Amber Hawksley* and Lauren Farber
}

\section{Introduction}

Accurate clerking is vital in ensuring optimal management of medical patients. Previous studies have highlighted the importance of pro forma use in maintaining documentation standards. This study aimed to refine an existing clerking pro forma and explored the subsequent impact of transition to electronic patient records (EPR).

\section{Materials and methods}

A single centre retrospective analysis was conducted at Barnet General Hospital, Royal Free NHS Trust, over a year (JanuaryDecember 2018). All patients $(n=60)$ were admitted during medical take to the acute or short stay units. Three groups of documentation were audited, 20 patients in each: clerking using no existing pro forma; introduction of a standardised clerking pro forma and post introduction of EPR. Comparison of medical clerking standard was made against guidelines outlined by the professional record standards body (PRSB). ${ }^{1}$ Adherence to highlighted domains was documented.

\section{Results and discussion}

Across all three groups, patient demographics and admission details and common sections of a history (presenting/history of complaint and past history) were documented for all patients. This was also the case for cardiorespiratory and abdominal examinations.

With regards to history taking, introduction of the pro forma led to improved documentation of review of systems ( 25 to $45 \%$ ). Additionally, social circumstances were better explored; living situation ( 45 to $75 \%$ ); occupation ( 15 to $50 \%$ ), smoking and alcohol history (50 to $80 \%$ and 45 to $70 \%$ respectively) and independence in activities of daily living ( 40 to $70 \%$ ). Though least documented, neurological examination documentation improved ( 45 to $70 \%$ ).

Clerking standards were generally maintained during transition to EPR. Further improvements from the introduction of a pro forma were seen, particularly in drug allergy histories (75 to 100\%) and their severity ( 43 to $100 \%$ ). All patients on EPR had investigations (blood tests, gases and scans) immediately uploaded when available.

Gradual improvement in resuscitation status was observed across all study groups (5, 50 and $100 \%$ respectively). However, treatment escalation plans remained variable (0,35 and $15 \%$ respectively).

Areas which remained poorly documented across all study groups included family history $(15,10$ and $25 \%$ respectively) as well as AMTS/4AT scoring ( $0 \%$ on EPR).

\section{Conclusion}

Introduction of a medical clerking pro forma has led to sustained improvements in domains of medical clerking documentation. Although the introduction of EPR can be daunting, provided there is adequate support and training for staff, benefits can be observed. There is scope for introduction and re-auditing of an updated pro forma in the future.

\section{Reference}

1 Body Professional Record Standards. Standard for the structure and content of health and care records. London: PRSB, 2018. 